\title{
El arte del consensus en Europa: ¿es aplicable la gobernanza de la Unión Europea universalmente?
}

\author{
Yolanda Gamarra Chopo* \\ Profesora Titular de Derecho internacional público y Relaciones internacionales \\ Universidad de Zaragoza
}

Recibido: 01.10.2012

Aceptado: 15.10.2012

\begin{abstract}
Resumen. Este estudio trata de la experiencia constitucional de la Unión Europea (UE) desde una perspectiva global recurriendo al «modelo de gobernanza» de la política exterior europea. Dos cuestiones centrales articulan el estudio: 1) si el «modelo de gobernanza» de la UE es aplicable universalmente, y 2) si la experiencia constitucional de la UE resulta eficaz desde una perspectiva global. Para alcanzar tales objetivos el estudio se divide en tres partes. Una primera, trata del «modelo de gobernanza» de la política exterior de la UE como poder normativo. Una segunda, evalúa la evolución de la política exterior de la UE desde su creación en la década de los años cincuenta. Y una tercera, explora, a través de tres ilustraciones, la aplicación del «modelo de gobernanza» de la UE, y su eficacia. El estudio concluye que el carácter distintivo de la UE, como actor global, no es tanto por la promoción de objetivos, principios y valores y/o intereses, o el recurso al derecho blando -soft-law- frente al derecho duro - hard-law-, sino más bien por el «modelo de gobernanza» de la política exterior que promueve. En este contexto afloran dos cuestiones conexas: 1) si el modelo de gobernanza de la UE en su política exterior es más eficaz cuando se enfrenta a problemas colectivos que el promovido por otros actores, y 2) si el «modelo de gobernanza» que aplica y promueve la UE es realmente colectivo y participatorio, o se trata más bien de un instrumento que utiliza la UE para exportar sus reglas sustantivas y promover sus intereses particulares, sin tener en cuenta las particularidades de los otros actores.
\end{abstract}

Palabras Claves: Gobernanza Global, Política Exterior de la Unión Europea, Soberanía, Derecho Global, Constitucionalismo, Pluralismo.

Abstract. This study addresses the constitutional experience of the European Union (EU) from a global perspective with respect to the "model of governance» of European foreign policy. Two principle issues are discussed: 1) whether the «model of governance» of the EU is universally applicable and 2) whether the constitutional experience of the EU is effective from a global viewpoint. The study is divided into three parts. The first deals with the «model of governance» of EU foreign policy as a

*Visiting Researcher at the Institute for Global Law and Policy, Harvard Law School, Harvard University, Boston, Mass., y Fellow at the Royal Complutense College at Harvard University, 2012. Esta actividad se enmarca en el programa de movilidad de investigación del profesorado del Ministerio de Educación (BOE, 12 de enero de 2012), y del Gobierno de Aragón (BOA, 1 de diciembre de 2011), y del Grupo de Investigación del Gobierno de Aragón (S 102), «Nuevas vías de participación política en democracies avanzadas». Gracias a Sandra Vicente e Ignacio de la Rasilla por su constante apoyo en la tarea investigadora. 
«normative power». The second assesses the evolution of EU foreign policy from its beginnings in the 1950s. The third explores, by means of three illustrations, the application of the EU «model of governance» and its effectiveness. The study concludes that the distinctive character of the EU as a global actor is not defined so much by its promotion of objectives, principles, and values and/or interests, or its recourse to soft law as opposed to hard law, as by the «model of governance» of its foreign policy. This gives rise to two related questions: 1) whether the «model of governance» of the EU in its foreign policy is more effective when faced with collective problems than that developed by other actors and 2) whether the «model of governance» developed and applied by the EU is truly collective and participatory, or an instrument used by the EU to export its substantive laws and to promote its own particular interests without taking into account the particular interests of other actors.

Key Words. Global Governance, European Union Foreign Policy, Sovereignty, Global Law, Constitutionalism, Pluralism.

Sumario: 1. Introducción.-2. Distinguiendo el modelo de gobernanza europea como "poder normativo".-3. De 1951 a 2007: una aproximación a la política exterior de la UE.-4. Explorando el «modelo de gobernanza» de la UE en sus relaciones internacionales en tres ilustraciones.-4.1. La cooperación UE-América Latina.-4.2. La política de la UE hacia la Corte Penal Internacional.-4.3. El marco cooperativo de la UE en materia de seguridad y defensa.-5. Conclusiones.

"Europe is not an actor in International affairs nor does it seem likely to become one. The Europe with which I am concernid is the actual one of state governments in which a minor role is placed, chiefly as instruments of cooperation between governmnets, by varoius committiees, assemblies and secretariats bearing the designation European, inlcuiding those of the Eurpean Community amongst others» (H. Bull «Civilian power Europe: A Contradiction in Terms», Journal of Common Market Studies, 1982, vol. 21, p. 151)

\section{INTRODUCCIÓN}

De entre las discusiones existentes en torno a la problemática de la gobernanza mundial, una de las escuelas de pensamiento defiende la idea de un cambio en las lógicas interestatales clásicas en cuanto a una sustitución del poder de elección monopolística e irreversible del Estado-nación (propia de la visión de Estados Unidos de Norteamérica), por la norma estable, colectiva y negociada, y soberanías compartidas (desarrollada por los Estados europeos en el marco de la UE) ${ }^{1}$. La lógica europea pretende, a través de las lógicas de gobernanza mundial, reducir la estructura «anárquica» del sistema interna-

${ }^{1}$ WeILER, J., «European Neo-Constitutionalism: in Search of Foundations for the European Constitutional Order», Bellamy, R. y Castiglione, D., Constitutionalism in Transformation: European and Theorical Perspectives, Blackwell, Oxford, 1996, pp. 105-121. 
cional. Esta filosofía de las normas tiene por objeto crear un escenario donde los protagonistas del sistema internacional estén encuadrados en una malla normativa a la vez densa y, si es posible, coercitiva. Esto es posible, en el caso de la UE, por el poder y el lugar que ocupa el TJUE como garante del sistema.

Los pilares de la renovación del constitutionalismo internacional o mundial son variados e incluyen el auge del proceso de constitucionalismo europeo y su modelo de «constitucionalismo a múltiples niveles» ${ }^{2}$, así como el efecto que éste último tiene con otros procesos de integración regional, la extensión y revitalización del modelo constitucional liberal doméstico tras el final de la Guerra Fría y el consecuente desarrollo de un mayor interés por el constitucionalismo internacional comparado ${ }^{3} \mathrm{y}$, aún, como corolario, por el Derecho internacional comparado 4 .

El diálogo entre tribunales es una realidad global que va más allá de las fronteras del sistema constitucional comunitario. Los juristas nacionales recurren cada vez con mayor asiduidad al derecho comparado para resolver problemas que se plantean en el interior de sus ordenamientos. La sociedad global es una constante fuente de exigencias por la creciente movilidad de las personas, y por los constantes incrementos de los intercambios económicos. De la misma manera que la redacción de cartas de derechos humanos regionales e internacionales, y la progresiva articulación de legislaciones comerciales vinculadas con la liberalización de las economías y la expansión del derecho internacional privado ha propiciado la emergencia de un acervo jurídico global a disposición de las diversas cortes constitucionales. Las políticas exteriores y de seguridad de los Estados, en el contexto de la UE, se asientan en principios cada vez más convergentes. Y la moneda colectiva europea, el euro, con sus propias ambivalencias, es ya una moneda de referencia global ${ }^{5}$.

Este estudio revisita, como punto de partida, una cuestión familiar como es la naturaleza de la «política exterior» de la UE. El estudio comienza con el análisis de las posiciones existentes en cuanto a la naturaleza de la UE como actor internacional, y si ésta se fundamenta en un conjunto

2 Pernice, I., «The Treaty of Lisbon: Multilevel Constituionalism in Action», Columbia Journal of European Law, 2009/3, vol. 15, pp. 349- 407.

3 Tushnet, M., «Comparative Constitutional Law», Reimann, M. y Zimmerman, R. (eds.), The Oxford Handbook of International Law, Oxford University Press, 2006, pp. 1225-1257.

${ }^{4}$ Koskenniemi, M., «The Case for Comparative International Law», Finnish Yearbook of International Law, 2011, pp. 3 et seq.

${ }^{5}$ Gamarra, Y., «Efectos de las crisis globales sobre el sistema monetario internacional: ¿una moneda de reserva alternativa al dólar?», MARTín Y PÉREZ DE NANCLARES, J. (Coord.), Estados y organizaciones internacionales ante las nuevas crisis globales. XXIII Jornadas ordinarias de la Asociación Española de Profesores de Derecho internacional y Relaciones internacionales-AEPDIRI-celebradas en La Rioja el 10, 11 y 12 de septiembre de 2009), AEPDIRI, Iustel, Universidad de La Rioja, 2010, pp. 611-622. 
de valores, principios y normas, y/o en sus propios intereses ${ }^{6}$. Dejando a un lado la distinción entre la promoción de valores y/o consecución de intereses de la UE, el análisis se centra en el «modelo de gobernanza» ${ }^{7}$ de la política exterior desde una posición no europea, sobre todo su tendencia a actuar colectivamente más que unilateralmente, y a establecer e institucionalizar el modelo de gobernanza en un amplio espectro de materias. La primera parte trata del modelo de gobernanza de la política exterior de la UE. La segunda de la evolución de la política exterior. La tercera propone una serie de ejemplos de «modelo de gobernanza» en las relaciones internacionales de la UE. El estudio termina con la exposición de una serie de cuestiones sobre el impacto del «modelo de gobernanza» de la política exterior de la UE.

\section{DISTINGUIENDO EL MODELO DE GOBERNANZA EUROPEA COMO «PODER NORMATIVO»}

Hay una variada y rica literatura de las relaciones internacionales de la UE como actor global ${ }^{8}$. Esta literatura de diferente manera se pregunta por el carácter del poder externo y político de la UE, frecuentemente distinguiéndola de la política exterior de los Estados, y algunas veces se compara, implícita o explícitamente, con el funcionamiento de Estados Unidos de Norteamérica. Una de las tesis más populares es la de considerar a la UE como «poder normativo». No obstante, la noción de poder normativo en sí misma es contestada, esto es, es utilizada para referirse ampliamente a la "promoción de normas en un sentido normativo" de la UE, por ejemplo, en la promoción del multilateralismo y de valores como el respeto del derecho internacional, los derechos humanos y la democracia, a través de métodos no coercitivos ${ }^{9}$. Un

\footnotetext{
${ }^{6}$ Keohane, R., «Governance in a Partially Globalized World»,American Political Science Review, 2001, 95, pp. 113. Sobre la necesaria coexistencia de normas y valores con las instituciones de gobernanza. En cuanto a la posición del Tribunal de Justicia de la UE en relación con la vinculación de la UE a los valores recogido en el artículo 3(5) TEU, vid. C366/10, ATAA $v$ Secretary of State for Climate Change, Judgment of 21 December 2011, par. 101.

${ }^{7}$ En el sentido expuesto por BÚRCA, G. DE, «EU External Relations: the Governance Mode of Foreign Policy», Blockmans, S. et al., The Legal Dimension of Global Governance: What Role for the EU, Oxford University Press, 2012.

${ }^{8}$ En buena medida esa literatura está escrita por europeos, entre otros, GERRITS, A. (ed), Normative Power Europe in a Changing World: A Discussion, 2009, disponible en: $<$ http://www.clingendael.nl/publications/2009/20091200_cesp_paper_gerrits.pdf $>$ (última consulta: 05.10.2012), y Sicurelli, D., The EU's Africa Policy: Norms, Interests and Impact, 2010, y LuCARelli, S. y Fioramonti, L. (eds), External Perceptions of the European Union as a Global Actor, 2009.

${ }^{9}$ MANNERS, I., «Normative power Europe: a contradiction in terms? », Journal of Common Market Studies, 2002, vol. 40, no 2, pp. 235-258; SJURSEn, H., «The EU as a normative power: How can this be?», Journal of European Public Policy, 2006, vol. 13,
} 
término utilizado para describir el papel externo de la UE es el de poder soft ${ }^{10}$, así como poder estructural que influye en estructuras y normas globales ${ }^{11}$.

La UE es considerada, a su vez, como un poder civil, en oposición al poder militar ${ }^{12}$, mientras otros han enfatizado su poder civilizador, en una adaptación de la idea sociológica de Norbert Elias del proceso civilizador ${ }^{13}$. La UE también ha sido considerada como poder global ${ }^{14}$, sistema de seguri$\mathrm{dad}^{15}$, imperio ${ }^{16}$, imperio normativo ${ }^{17}$ y hegemón normativo regional ${ }^{18}$.

Cada una de estas aproximaciones, diferentes pero relacionadas entre sí, tratan del carácter diferenciador de la política exterior de la UE, enfatizando

pp. 235-251, y una posición crítica. en DíEz, T., «Constructing the Self and Changing Others: Reconsidering Normative Power Europe», Millenium Journal of International Studies, vol. 33, 2005, pp. 613-636. Así como, el estudio de Whitman, R. (ed), Normative Power Europe: Empirical and Theoretical Perspectives, Palgrave, 2011. Una reciente defensa del término como un modelo ideal en ForsBERG, T., «Normative Power Europe, Once Again: A Conceptual Analysis of an Idealtype», Journal of Common Market Studies, 2011, vol. 49, pp. 1183-1204.

${ }^{10}$ Nye, J., Soft Power: The Means to Success in World Politics, Public Affairs, 2004. Del mismo autor, The Future of Power, Public Affairs, 2011, en el que define el poder «suave» (smart power) como una habilidad para combinar el poder «duro» (hard power) y el poder «blando» (soft power) en una estrategia efectiva. También, KaGAN, R., Of Paradise and Power, America and Europe in the New World Order, Knopf, 2003.

${ }^{11}$ Barnett, M. y Duvall, R., Power and Global Governance, CUP, 2004, Capítulo1, y Keukeleire, S. y Macnaughtan, J., The Foreign Policy of the European Union, Palgrave, Macmillan, Londres, 2008.

${ }^{12}$ DuCHÊNE, F., «The European Community and the uncertainties of interdependence», Kohnstamm, M. y Hager, W. (eds) A Nation Write Large? Foreign Policy Problems before the European Community, Macmillan, Basingstoke 1973, y TĖLo, M., Europe: A Civilian Power?, Palgrave, Macmillan, 2006. Una aproximación crítica al debate europeo sobre el poder «civil»y «normativo» en ORBIE, J., «Current Debates», Cooperation and Conflict, 2006, vol. 40, p. 123 et seq.

${ }^{13}$ Linklater, A., «A European Civilizing Process», Hill, C. y SMith, M., International Relations and the European Union, OUP, 2005.

${ }^{14}$ Bretherton, C. y Vogler, J., The European Union as a Global Actor, Routledge, 2nd ed, 2006, y Rogers, J., «From Civilian Power to Global Power: Explicating the EU's Grand Strategy through the use of Discourse Theory» Journal of Common Market Studies, vol. 47, 2009, pp. 831-862. Un interesante debate focalizado en la política ética de la UE como elemento distintivo en Howse, R. y NiCOLAIDIS, K., "This is my EUtopia», Journal of Common Market Studies, vol. 40, 2002, pp. 767-792.

${ }^{15}$ Charillon, F., «The EU as a Security Regime», European Foreign Affairs Review, 10, 2005, pp. 517-533.

${ }^{16}$ ZIELONKA, J., Europe as Empire: The Nature of the Enlarged European Union, OUP, 2006.

${ }^{17}$ LAIDI, Z., «The Unintended Consequences of European Power», Les Cahiers européens de Sciences Po, No. 5/2007.

${ }^{18}$ HaukKala, H., «The EU as a Regional Normative Hegemon, The Case of European Neighborhood Policy», Whitman, R. G. (ed.), Normative Power Europe: Empirical and Theoretical Perspectives, Palgrave Macmillan, Basingstoke \& New York, 2011, p. 4564. 
sus recursos, sus objetivos, los medios para alcanzar sus objetivos, o la combinación de los tres. El argumento que pretendo articular comparte premisas de esta literatura, pero prestando especial atención al carácter de las relaciones internacionales de la UE que difiere de la existente consideración normativa o civil. A la hora de describir a la UE como «modelo de gobernanza» de la política exterior, los temas en los que me centro afectan: primero, a la preferencia de la UE por la cooperación estable e institucionalizada con Estados y otras organizaciones internacionales, y segundo, al uso frecuente de marcos reguladores que afectan a objetivos comunes, y a la coordinación de la política exterior como si fuera política interna. Esta preferencia parece ser parte de un producto de externalización de las prácticas de la UE, las cuales son desarrolladas internamente como parte de su proyecto original de integración transnacional europeo. No obstante, la elección de la estrategia externa de la UE es consecuencia del entorno global en el que actúa y proyecta sus valores e intereses ${ }^{19}$.

Definir con mayor precisión el «modelo de gobernanza» de la política exterior de la UE requiere tener en consideración que la UE persigue una política exterior liberal, en términos generales. Sin duda, la UE conduce sus relaciones exteriores de una manera particular en relación a cómo las articulan otros actores internacionales, al mismo tiempo que busca un elemento diferenciador. En esta senda, dos ejes articulan el estudio: primero, ¿qué objetivos son perseguidos? y, segundo, ¿de qué modo son perseguidos? Los diferentes objetivos perseguidos por la UE en su política exterior se asienta en dos premisas. La primera consiste en demostrar cómo la UE, en la búsqueda de sus objetivos, actúa, en general, colectivamente -algunas veces multilateralmente, si bien no siempre-, más que unilateralmente ${ }^{20}$. Ese modo de actuar abarca tanto los medios como los objetivos.

La segunda trata de demostrar cómo la normativa de la UE, en la búsqueda de los objetivos por medios colectivos, externaliza el «modelo de gobernanza» desarrollado en sus políticas internas. Así, la UE trata de crear e institucionalizar relaciones con otros Estados y órganos, y establecer un marco regulador transnacional y amplio para coordinar y gobernar varias áreas temáticas, a menudo enfatizando objetivos, a largo más que a corto plazo, y persiguiendo estrategias proactivas y/o preventivas más que reactivas. Mientras los tradicionales o típicos instrumentos de las relaciones exteriores provienen de la práctica diplomática, bien a través del recurso a incentivos o sanciones económicas y políticas de variado tipo, bien por el uso del poder militar y la fuerza, el «modelo de gobernanza» de la política exterior de la UE se centra en el establecimiento de una red de relaciones estables, institucionalizadas con otros Estados, órganos, regiones,

${ }^{19}$ Dunof J. L. y Trachtman, J. P. (eds.), Ruling the World: Constitutionalism, International Law and Global Governance, Cambridge University Press, 2009.

${ }^{20}$ La preferencia por la aproximación colectiva es recogida en el artículo 3 par. 5 del TUE, así como en documentos de la propia estrategia de la UE. 
las cuales llegan a crear otras instituciones de policy-making, norm-generating, coordinación y control, y que, en buena medida, son el reflejo del sistema normativo interno desarrollado por la UE. Más aún, mientras la política exterior convencional ha sido considerada como una política autónoma formulada y desarrollada por actores estatales con el fin de perseguir intereses nacionales asentada en específicas más que difusas maneras de reciprocidad, el «modelo de gobernanza» de la política exterior de la UE promueve la intensificación de la interdependencia e institucionalización, y se asienta en el establecimiento de una difusa, más que específica, forma de reciprocidad ${ }^{21}$. Finalmente, los compromisos alcanzados en marcos en los que se ha puesto en práctica la gobernanza externa de la UE frecuentemente persiguen múltiples objetivos, combinando e/o integrando diferentes objetivos e intereses.

La evolución de las relaciones exteriores de la Comunidad Europea y de la UE en el tiempo pueden ofrecernos un número elevado de razones para el desarrollo del carácter distintivo del «modelo de gobernanza» de la política exterior. Este recorrido quizás nos ayude a entender la especificidad del «modelo de gobernanza» de la UE, en el caso particular de la política exterior. De ello tratamos en el siguiente epígrafe.

\section{DE 1951 A 2007: UNAAPROXIMACIÓN A LA POLÍTICA EXTERIOR DE LA UE}

En las dos últimas décadas, la UE ha reforzado su papel de actor global $\mathrm{y}$ ha incrementado su presencia y visibilidad en la esfera internacional ${ }^{22}$. En la década de los ochenta, Hedley Bull escribió: no hay una comunidad supranacional en el occidente europeo, tan sólo un grupo de estados-nación ${ }^{23}$. En estos momentos, hay incluso cierto debate, con menor insistencia que el provocado por $\mathrm{H}$. Bull hace treinta años, acerca de si realmente existe la «política exterior» de la $\mathrm{UE}^{24}$. De un lado, se encuentran aquellos que ven a la UE como poco más que una mera correa de transmisión de la política exterior de los Estados más fuertes ${ }^{25}$. De otro, aquellos que ven a la UE como una su-

\footnotetext{
${ }^{21}$ Keohane, R., «Governance in a Partially Globalized World», American Political Science Review, cit., p. 4.

${ }^{22}$ Cremona, M. (ed.), Developments in EU External Relations Law, Cambridge University Press, 2008.

${ }^{23}$ Bull, H., «Civilian Power Europe: A Contradiction in Terms? », Journal of Common Market Studies, 1982, vol. 21, p. 151.

${ }^{24}$ Peterson, J., «Trade Policy as Foreign Policy», The European Union Studies Association (EUSA), Montreal, 2007, y WhITE, B. Understanding EU Foreign Policy, Palgrave, 2001, pp. 36-37.

${ }^{25}$ TorreblancA, J. I., «Sarkozy's Foreign Policy: Where do European Interests and Values Stand?», European Council on Foreign Relations, Febrero de 2008, disponible en www.ecfr.eu/page//documents/Torreblanca SarkozyForeignPolicy.pdf (última consulta: 25.09.2012).
} 
perpotencia global emergente por sí misma ${ }^{26}$. En una posición intermedia, diferentes visiones afloran acerca de la naturaleza y carácter de la UE como actor global y su conducta en las relaciones internacionales ${ }^{27}$.

Sin embargo, resulta indudable que desde la creación de la UE con la instauración del segundo pilar de la «política exterior y de seguridad común» en el Tratado de la UE, coloquialmente conocido como Tratado de Maastricht (1992), la UE y sus Estados miembros han tomado conciencia de la necesidad de adoptar una serie de pasos para formalizar e institucionalizar las relaciones internacionales. El más reciente de los pasos dados se encuentra en el Tratado de Lisboa, de 2007, en adelante, TUE (2007), al crear una serie de instituciones de política exterior con el propósito de visibilizar y dotar de coherencia a las relaciones internacionales de la $\mathrm{UE}^{28}$. En otras palabras, la UE y sus Estados miembros al revisar los tratados están constantemente reforzando su perfil internacional y potenciando su carácter de actor global.

La UE (anteriormente, la CE) mantuvo relaciones internacionales desde el momento de su creación en los años cincuenta, en particular, en el desarrollo de relaciones comerciales y de cooperación. El comercio internacional -la política comercial común- fue una de las pocas áreas de competencia externa explícita reconocida en el Tratado de la Comunidad Económica Europea (CEE), de 1957. De otro lado, el desarrollo de la política exterior emergió a través de los acuerdos de «cooperación» $\mathrm{y}$ «asociación» con un amplio grupo de países, en particular con las antiguas colonias de los Estados miembros, más concretamente se articuló por medio de los Acuerdos de Yaoundé, Lomé y Cotonou con los Estados Africanos, Caribeños y del Pacífico (Países ACP). Las relaciones externas de la CEE también afectaron a otras áreas como el transporte ${ }^{29}$, pesquerías, y política medioambiental ${ }^{30}$.

En esta senda, el término «relaciones exteriores» viene a tener un específico significado en el marco de la UE. El término es usado para identificar distintas actividades en el campo del comercio, ayuda a la cooperación, y un número de áreas sectoriales de la CEE, en contraste con la específica dimensión política de la «política exterior» de la UE. «Relaciones Exteriores»abarca

${ }^{26}$ McCORMick, J., The European Superpower, Palgrave, Macmillan 2006, y MorAVCSIK, A., "Europe: Quietly Rising Superpower in a Bipolar World" (estudio sin publicar), 2009.

${ }^{27}$ White, B., «Foreign Policy Analysis and European Foreign Policy», TonRA, B. y Christiansen, T. (eds) Rethinking European Union Foreign Policy. Un análisis anterior en Zielonka, J. (ed), Paradoxes of European Foreign Policy, Kluwer, 1998.

${ }^{28}$ BÚRCA, G. DE, «EU External Relations: the Governance Mode of Foreign Policy», cit. supra nota 6.

${ }^{29}$ Macleod, I., Hendry, I. D. y Hyett, S., The External Relations of the European Communities: A Manual of Law and Practice, OUP, 1996, Cap. 11, y MenciK VAN ZEBINSKY, A., External Relations in Air Transport, Kluwer, 1996.

${ }^{30}$ Mastellone, C., «The External Relations of the EEC in the Field of Environmental Protection», ICLQ, 1981, vol. 30, pp. 104417. 
áreas llamadas de «low politics» en las que la UE es propensa a comprometerse, mientras las materias de «high politics» afectan a los asuntos tratados en foros intergubernamentales por los Jefes de Estado y de Gobierno en el marco de la Cooperación Política Europea (CPE), desde los años setenta. El artículo 30 del Acta Única Europea (1986) formalizó la CPE, y más tarde sirvió de asidero a la Política Exterior y de Seguridad Común (PESC). Desde el Tratado de la UE (1992) hasta la actualidad ha habido avances cuantitativos y cualitativos hasta llegar a las provisiones del TUE (2007).

El TUE (2007) ha creado una serie de nuevas instituciones con la pretensión de dirigir a la UE en sus relaciones internacionales. Con el TUE (2007), los objetivos de la construcción europea resultan actualizados siendo necesario contribuir desde sus responsabilidades crecientes a la gestión de los problemas comunes de la globalización. El TUE facilita el liderazgo de la UE en la esfera internacional a través de la profundización de su propio modelo. Como también, por primera vez, se formula un marco normativo para la definición de la política exterior europea, la política de responsabilidad basada en la ética, en la opción por la regulación internacional, el refuerzo de las instituciones internacionales y el multilateralismo. Se crean, además, nuevas instituciones e instrumentos para una mayor presencia internacional, entre los que destacan el Alto Representante de la Unión para los Asuntos Exteriores y la Política de Seguridad (al mismo tiempo que Vicepresidente de la Comisión), y establece el servicio diplomático (el Servicio Europeo de Acción Exterior), así como innovaciones en una Política de Defensa propia de una potencia civil ${ }^{31}$. El propósito de estos y otros cambios parecen responder a una más coherente y unificada política de relaciones internacionales de la UE.

Incluso, antes de los cambios institucionales del TUE (2007), varios movimientos dotaron de mayor coherencia a la UE en sus relaciones internacionales. El marco para las relaciones internacionales de la UE ha sido complejo y fragmentado en diferentes caminos, y no sólo en la separación de facto entre las supuestas políticas de alta o baja intensidad, sino más bien como resultado de la separación de esferas de la política de pilares de la UE introducida en el Tratado de Maastricht (1992), y compartiendo competencias entre la UE y los Estados miembros en distintos campos que tiene como resultado la noción de competencias «compartidas». La UE, empero, ha comenzado, de un lado, a desarrollar y utilizar una serie de estrategías, instrumentos y políticas para evitar las complejidades legales e institucionales de su cuarteado marco de relaciones internacionales, y de otro, ha avanzado en un modelo integrado. Tres ejemplos de esa estrategia integrada, instrumentos y políticas son, primero, la estrategia de ampliación y pre-adhesión que la UE desarrolló desde comienzos de 1999 con los países de la Europa Central y Oriental (PECO's);

${ }^{31}$ Gutiérrez Espada, C. y Cervell Hortal, Mª J., La adaptación al tratado de Lisboa (2007) del sistema institucional decisorio de la Unión, su acción exterior y personalidad jurídica, 2010. 
segundo, la política de vecindad que fue lanzada en 2003, y tercero la política de interregionalismo perseguida por la UE en sus relaciones con otros grupos regionales ${ }^{32}$.

En suma, mientras la UE se mueve hacia un marco formalizado e integrado en sus relaciones exteriores, tanto en el campo de la política de seguridad y defensa, como en otros campos de la política exterior recogidos en el TUE (2007), la UE ha desarrollado una gobernanza de las relaciones internacionales en varias esferas, institucionalizando sus relaciones con otros Estados y entes, creando marcos específicos y procesos reguladores para alcanzar sus objetivos y buscando establecer una presencia particular en la escena internacional. Esta aproximación a la gobernanza parece construirse sobre los cimientos de la trayectoria de años de actividades desarrolladas por las instituciones de la CEE.

Hay una importante literatura que considera que la política exterior de los Estados está contagiada por las estructuras domésticas o política interna. La UE no es un Estado, no tiene una autoridad centralizada, ni instituciones similares a las de un Estado, en cambio, la gobernanza de la política exterior es, en parte, reflejo o extension de su manera de operar internamente. Resulta llamativo que la relación entre los modos internos de gobernar desarrollados por la UE y su emergente identidad de política internacional ha sido objeto reciente de interés por un creciente numero de autores de la $\mathrm{UE}^{33}$.

\footnotetext{
${ }^{32}$ Sobre este particular resulta de interés el estudio de SöDERBAUM, F. y LANGENHOV, L., "The EU as a Global Actor and The Role of Interregionalism: A Comparative Analysis», Journal of European Integration, 2005, Volume 27, nº 3, pp. 249-262. Así como ALEÇU DE Flers, N. y Regelsberger, E., «The EU and Interregional Cooperation», Hill, C. y SMith, M. (eds.), International Relations and the EU, Routledge, Londres/Nueva York, 2005, pp. 317-342; Hettne, B. y SöDerbaum, F., «Civilian Power or Soft Imperialism? The EU as a Global Actor and the Role of Interregionalism», European Foreign Affairs Review, 2005, vol. 10 pp. 535 552; Hardacre, A. y SMITH, M., «The EU and the Diplomacy of Complex Interregionalism», Hague Journal of Diplomacy, 2009, n 4, p. 197, y Aggerwal, V., «Explaining Trends in EU Interregionalism», AgGarwal, V. y FogarTy, E. (eds.), European Union Trade Strategies: Between Globalism and Regionalism, Palgrave, Londres, 2004.

${ }^{33}$ FARRELL, M., «EU External Relations: Exporting the EU Model of Governance?», European Foreign Affairs Review, vol. 10, 2005, pp. 451-462. Así como, los estudios de SCHIMMELPFENNIG, F. Y WAGNER, W., «External governance in the European Union», Journal of European Public Policy, vol. 11, 2004, pp. 657-660; LAVEnEX, S., «EU External Governance in Wider Europe», JEPP, vol. 11, 2004, pp. 680-700; RosAMUND, B., «The EU model of governance in world politics», EFAR, vol. 10, 2005, pp. 463-478; WARLEIGH, A., «Learning from Europe: EU Studies and the Rethinking of International Relations» European Journal of International Relations, $\mathrm{n}^{\circ}$ 21, 2006, pp. 31-51; MYRJORD, A., "Governance Beyond the Union: EU Boundaries in the Barents EuroArctic Region», European Foreign Affairs Review, vol. 8, 2003, pp. 239-257; FiltenborG, M. S., GÄnZle, S. y Johannsen, E., «An Alternative Theoretical Approach to EU Foreign Policy: «Network Governance» and
} 
La gobernanza de la UE puede ser explicada al menos por dos razones. Primero, su identidad: la UE es un actor sui generis basado en su historia, evolución, y su modo de operar internamente, que tiende a proyectar -esa identidad y modelo de operar- en sus relaciones exteriores. Segundo, obedece a una cuestión instrumental. La UE ha gozado de éxito (hasta el estallido de la crisis de identidad por la crisis financiera y económica, crisis soberana de 2007) como sistema económico y politico regional, y ha encontrado caminos de gobernar sus problemas internos reconciliando la soberanía de los Estados miembros y los objetivos comunes gestionando bien la interdependencia creciente en la esfera internacional.

Ese «modelo de gobernanza» se proyecta en las relaciones con otros actores para estrechar la cooperación entre otros sistemas regionales ${ }^{34}$. La gobernanza interna se reproduce en las relaciones internacionales que mantiene con diferentes Estados y actores internacionales. La gobernanza de la UE también está condicionada por el marco global, y por los intereses de nuevos actores globales emergentes ${ }^{35}$.

\section{EXPLORANDO EL «MODELO DE GOBERNANZA» DE LA UE EN SUS RELACIONES INTERNACIONALES EN TRES ILUSTRACIO- NES}

\subsection{La cooperación UE-América Latina}

La cooperación desarrollada entre la UE y América Latina, todavía escasa, es uno de los supuestos en los que hay que detenerse para estudiar si el «modelo de gobernanza» de la UE es aplicable universalmente. La VI Cumbre UE-América Latina y el Caribe, celebrada en mayo de 2010, a pesar de las dudas despertadas acerca de su alcance, supuso un paso cualitativo y cuantitativo en la consolidación de las relaciones de la UE con la región latinoamericana y caribeña.

the Case of the Northern Dimension Initiative», Cooperation and Conflict, vol. 37, 2002, p. 387; DíEZ, T., «The Imposition of Governance: Transforming Foreign Policy Through EU Enlargement», Copenhagen Peace Research Institute Working Paper, (2000), y KeUKELEIRE, S. y Schunz, S., Foreign Policy, Globalization and Global Governance: The European Unio$n$ 's Structural Foreign Policy, 2008, disponible en <http://www.jhubc.it/ecpr-riga/ virtualpaperroom/102.pdf $>$ (última consulta: 24.09.2012).

34 De Lombaerde, P., Söderbaum, F., Langenhove, L. Van y Baert, F., «The Problem of Comparison in Comparative Regionalism», University of Florida-Jean Monnet/Robert Schuman Paper Series, Vol. 9, No 7, 2009, pp. 1-22.

${ }^{35}$ Rosamond, B., «The EU model of governance in world politics», European Foreign Affairs Review, vol. 10, 2005, pp. 463-478, ha defendido que «there is no single EU mode of governance and, therefore, that the external projection of the EU's governance identity is far from straightforward. Moreover, in terms of governance models, the EU does not simply practice a weird, internallygenerated, path dependent mode, but rather its governance practice is part of a broader global exchange of governance methodologies». 
Los cambios en Europa y América Latina han alterado radicalmente, en la primera década del siglo XXI, el mapa de las relaciones interregionales, así como las prioridades de las dos regiones ${ }^{36}$. Un factor que no ha ayudado en las relaciones entre ambos bloques regionales es que América Latina no avanza en la integración, ni siquiera en el espacio latinoamericano, a pesar de la acumulación de nuevas instituciones. La superposición de iniciativas concurrentes no permite ni un proyecto, ni una interlocución válida con América Latina como región. A pesar de todo ello, existe un incremento del nacimiento de Sudamérica como actor internacional, liderado por Brasil. En realidad, América Latina no avanza en su dinamismo exterior, y Europa sí.

En las relaciones UE-América Latina, los intereses, y cada vez más las relaciones comerciales han distanciado a los bloques regionales, mientras que los valores y objetivos comunes las han acercado. La pérdida de competitividad de las economías de los Estados de la UE, y la fortaleza de los BRIC's son factores que ayudan a entender ese alejamiento. La VI Cumbre, en cambio, resolvió y encauzó gran parte del estancamiento en torno a la cooperación económica y comercial con los siguientes avances. En primer lugar, hay que citar la conclusión de las negociaciones para un acuerdo de Asociación con Centroamérica, como también, la conclusión del acuerdo comercial a varias bandas con Colombia y Perú. Asimismo, avanza el diálogo político y estratégico con Brasil (III Cumbre) y México (V Cumbre) y el bilateral con Chile (IV Cumbre). Se ha iniciado un diálogo político regional con el CARIFORUM, y quizás la gran noticia sea la reanudación de las negociaciones para un acuerdo comercial con MERCOSUR, que no sabemos en qué desembocará dadas las dificultades de esta organización, y la resistencia francesa por la cuestión agrícola.

La gran novedad de esta Cumbre residió en el refuerzo de ambas regiones como socios globales, esto es, una voluntad de entenderse para fijar posiciones comunes y crear sinergias en las instituciones internacionales con el objeto de abordar conjuntamente los problemas de la agenda internacional. Llama la atención, cuando menos, la coincidencia de los distintos socios en el diseño de una agenda común que incluye asuntos tales como la moratoria de la pena de muerte, el deseo de reforzar y ampliar la participación en la Corte Penal Internacional, la voluntad de regulación y supervisión económica y financiera internacional, en materia de cooperación para el desarrollo con la asunción de los compromisos de Monterrey, la necesidad de poner en marcha un diálogo global y nuevos acuerdos sobre cambio climático y biodiversidad,

${ }^{36}$ GrisAnt, L. X., El nuevo interregionalismo transatlántico: la asociación estratégica Unión Europea-América Latina, Documento de Divulgación IECI-04, BID, Buenos Aires, 2004. 
e incluso, de tratar de impulsar la reforma de los órganos de las Naciones Unidas, entre otros asuntos ${ }^{37}$.

En todos estos temas se tiene una visión común que a pesar de la densidad de la cooperación no se comparte con Estados Unidos de Norteamérica por su particular manera de entender y enfocar su papel en el mundo ${ }^{38}$. A partir de esta percepción común sobre la necesidad de profundizar en la gobernanza global a través de un multilateralismo eficaz, coloca a estos sesenta Estados en una posición privilegiada para incidir en las transformaciones necesarias de la sociedad internacional. La Asociación «UE-América Latina» la conforman un grupo de Estados que no sólo tiene una visión común sino que la articulan a través de una agenda operativa con el objetivo de alcanzar las transformaciones necesarias.

Además, para que la UE llegue a ser un actor mundial eficiente y dinámico, también tiene que situar la solidaridad en el centro del proyecto europeo. La solidaridad no es un derecho sin condiciones: depende de la responsabilidad tanto individual como colectiva. Como tal puede y debe conformar la toma de medidas y las relaciones de la UE en todos los niveles, entre individuos y organizaciones, y entre entes locales, regiones y Estados miembros. Así, podrá proyectar la idea de solidaridad europea a escala global y desarrollar con otros bloques regionales su «modelo de gobernanza» con el fin de demostrar su responsabilidad en la gestión de asuntos que afectan a todos por igual.

\subsection{La política de la UE hacia la Corte Penal Internacional}

La UE apoyó desde un primer momento el establecimiento y procedimiento de la Corte Penal Internacional (CPI), así como la creación y funcionamiento de otros tribunales internacionales tales como los tribunales penales ad hoc para la antigua Yugoslavia y Ruanda ${ }^{39}$.

La UE ha promovido y apoyado, incluso financieramente, a la CPI desde el mismo momento de su creación ${ }^{40}$. La UE adoptó una posición común y un Plan de Acción sobre las relaciones con la CPI y su política hacía la CPI in-

${ }^{37}$ GARcía CASAS, F., «Bicentenarios: en los umbrales de una nueva sociedad», GAMARRA, Y. (coord.), La idea de América en el pensamiento ius internacionalista del siglo XX, Zaragoza, Institución «Fernando el Católico», 2010, pp. 125 et seq.

${ }^{38}$ FabBrini, S. (ed.), The United States Contested. American Unilateralism and European Discounted, Routledge, Londres, 2006.

${ }^{39}$ Aoun, E., «The European Union and International Criminal Justice: Living up to its Normative Preferences», Journal of Common Market Studies, vol. 50, n 1, 2012, pp. 2136.

${ }^{40}$ Una evaluación de la consistencia y eficacia del apoyo de la UE a la CPI en MERTENS, M., «The International Criminal Court: A European Success Story?», LeCollege d'Europe, EU Diplomacy Papers 1/2011. 
ternamente coherente ${ }^{41}$. La UE ha utilizado una variedad de instrumentos para apoyar a la CPI, como presión diplomática sobre los Estados, asistencia técnica, económica y financiera a los Estados y ONG, e incluso ha llegado a incluir una cláusula en los acuerdos de comercio y cooperación ${ }^{42}$. El apoyo a la $\mathrm{CPI}$ es un buen ejemplo para justificar cómo la UE persigue los objetivos de estabilidad y paz internacionales por medio de la creación y funcionamiento de un régimen y una institución multilateral, y de cómo integra como suyos asuntos propios de la política internacional.

La posición de la UE hacia la CPI es utilizada, my frecuentemente, para contrastarla con la política de Estados Unidos de Norteamérica, quien se opuso a la creación de la CPI y llegó a tejer una red de acuerdos bilaterales para evitar la entrega de ciudadanos norteamericanos ante la $\mathrm{CPI}^{43}$. Mientras Estados Unidos de Norteamérica condiciona su apoyo a la CPI a la evaluación positiva de su Secretario de Estado, la UE prefiere la intervención de una institución internacional independiente. La relación del Consejo de Seguridad con la CPI explica, en buena medida, la política hostil de Estados Unidos de Norteamérica hacia la CPI. Antes y durante la Conferencia de Roma, la Administración Clinton pretendió establecer un control político sobre las actividades de la CPI, en especial cuando sus nacionales se viesen involucrados en investigaciones o enjuiciamientos ante la $\mathrm{CPI}^{44}$. En todo momento, esta Administración defendió que las actuaciones de la CPI requerían la aprobación del Consejo de Seguridad en relación con una situación englobada en el Capítulo VII de la Carta -al contar con el poder del veto-, así como la obligación de reconocer la competencia de la CPI antes de que ésta pueda iniciar algún tipo de acción ${ }^{45}$. Tales pretensiones no progresaron en las negociaciones con-

${ }^{41}$ Posición Común del Consejo 2003/444/CFSP, de 16 de junio de 2003. Así como una reciente decision del Consejo 2011/168/CFSP de 21 de marzo de 2011 sobre la CPI. También, el acuerdo entre la UE y la CPI de 2006 para la asistencia y cooperación entre ambas organizaciones: http://register.consilium.eu.int/pdf/en/05/st14/st14298.en05.pdf (última consulta: 2.09.2012).

${ }^{42}$ El ejemplo estandar es el article 11 del Acuerdo de Cotonu: «-In promoting the strengthening of peace and international justice, the Parties reaffirm their determination to: share experience in the adoption of legal adjustments required to allow for the ratification and implementation of the Rome Statute of the International Criminal Court; and ...fight against international crime in accordance with international law, giving due regard to the Rome Statute. The Parties shall seek to take steps towards ratifying and implementing the Rome Statute and related instruments.»

${ }^{43}$ Sobre esta política de USA hacia la CPI en Gamarra, Y., «La política hostil de Estados Unidos de Norteamérica contra la Corte Penal Internacional: los acuerdos bilaterales del artículo 98 (2) o la búsqueda de la impunidad», REDI, 2005, pp. 145-169.

${ }_{44}$ Acerca del papel de Estados Unidos en la Conferencia de Roma vid., Scheffer, D., «The United States and the International Criminal Court», AJIL, 1999, vol. 93, pp. 19 a 21.

${ }^{45} \mathrm{La}$ defensa del necesario control sobre la CPI se infiere de las palabras de R. Wedgwood, "(s)ome countries have instead supposed that the ICC and its Assembly of States Parties are entitled to mimic Security Council authority, unilaterally impleading 
virtiéndose en una de las primeras «deficiencias» del Estatuto denunciadas por las sucesivas Administraciones. Lejos de reconocerse la potestad exclusiva del Consejo de Seguridad para decidir en qué casos -incluidos en el Capítulo VII de la Carta-, la CPI puede iniciar investigaciones, la nueva CPI goza de carta blanca para actuar en las distintas situaciones ${ }^{46}$. No se necesita, así las cosas, la determinación previa por el Consejo de Seguridad para comenzar las investigaciones, por ejemplo, por agresión armada. No obstante, el Consejo de Seguridad está habilitado, primero y de acuerdo con el artículo 16 del Estatuto, para suspender las investigaciones o enjuiciamientos durante un año con posibilidad de renovación ${ }^{47} \mathrm{y}$, segundo y como contempla el artículo 13 párrafo b del Estatuto, para remitir una situación a la Fiscalía de la $\mathrm{CPI}^{48}$. Para la Administración de Estados Unidos de Norteamérica esa independencia de la CPI, así como su carácter permanente se tornan en factores impedientes de la aceptación del Estatuto de Roma.

La independencia de la CPI es interpretada como un debilitamiento del Consejo de Seguridad en sus funciones de mantenimiento de la paz y seguridad internacionales ${ }^{49}$. Pese a esta pesimista e interesada construcción por parte de Estados Unidos de Norteamérica, buen número de observadores acreditados en la Conferencia de Roma coincidieron en asegurar que sobre el Consejo de Seguridad recae la potestad de determinar la existencia de una amenaza a la paz, de la misma manera que el artículo 5 párrafo 2 del Estatuto hace referencia a su compatibilidad con las disposiciones de la Carta de Naciones Unidas $^{50}$. Esta visión del debilitamiento del máximo órgano garante de la paz y

the nationals of states that have declined to join the Court. Washington sees this as a usurpation of the Security Council's established position in international law and in the architecture of the UN Charter. Under the Charter, the permanent members of the Security Council must concur in any UN enforcement decisions», WEDGWOOD, R., «The United States and the International Criminal Court: The Irresolution of Rome», Law and Contemporary Problems, 2001, pp. 198 y 199.

${ }^{46}$ Limitadas a las condiciones establecidas en el Estatuto. Una visión crítica en GuTiÉRREZ ESPADA, C., «La Corte Penal Internacional (CPI) y las Naciones Unidas. La discutida posición del Consejo de Seguridad», $A D I, 2002$, pp. 60 y ss.

${ }^{47}$ Como se presentó en las polémicas resoluciones 1422 (2002) y 1487 (2003) del Consejo de Seguridad de Naciones Unidas.

${ }^{48}$ Caso de los crímenes cometidos en Sudán (Darfur) recogido en la resolución 1593 (2005) del Consejo de Seguridad, de 31 de marzo, KIRGIS, F. L., «UN Commission's Report on Violations of International Humanitarian Law in Darfur Security Council Referral to the International Criminal Court», ASIL Insight, 5 de abril de 2005, disponible en $<$ http://www.asil.org/insights/2005/02/insight050204a.html > (última consulta: 24.09.2012).

${ }^{49}$ Statement of the United States Delegation to the Preparatory Committee on the Establishment of an International Criminal Court, March 23, 1998, y WelleR, M. «Undoing the global constitution: UN Security Council action on the International Criminal Court», International Affairs, vol. 78/4, 2002, pp. 703 y 704.

${ }^{50}$ SAdat, L. N. y CARden, S. R., «The New International Criminal Court: An Uneasy Revolution», The Georgetown Law Journal, vol. 88, 2000, p. 450. 
seguridad internacionales fue confirmada años más tarde por el representante de la Administración Bush ante el Consejo de Seguridad al afirmar que «no ceden a ningún país su liderazgo en la lucha por la justicia internacional y la rendición de cuentas en materia de crímenes de guerra. Al fin y al cabo, los Estados Unidos fueron el primer país en codificar el derecho de la guerra y el derecho internacional humanitario (...) Han sido y seguirán siendo enérgicos defensores de los tribunales establecidos bajo la égida del Consejo de Seguridad. No obstante, a diferencia de la CPI, esos tribunales deben rendir cuentas al Consejo de Seguridad $»^{51}$. La rendición de cuentas de la CPI es después de todo lo que buscó y busca la Administración de Estados Unidos de Norteamérica. Cualquier supervisión independiente de sus acciones (o de las de sus oficiales y soldados) entrañaría demasiado riesgo; riesgo de decisiones políticas que podrían ser incorrectas o injustas, pero también riesgo de decisiones correctas y justificables que, por el contrario, chocarían con el percibido interés político o geopolítico de Estados Unidos de Norteamérica en un momento dado.

La UE y sus Estados miembros apostaron por una CPI sólida y eficaz en la acción de prevenir la impunidad ${ }^{52}$. La UE es el actor de la esfera internacional con el poder político, instrumentos jurídicos y medios financieros para materializar este proyecto, junto con Estados afines, y otros actores internacionales.

\subsection{EI marco cooperativo de la UE en materia de seguridad y defensa}

Las primeras experiencias de las fuerzas operativas militares europeas con la alianza transatlántica tuvieron lugar dentro del marco cooperativo de la UEO y la OTAN ${ }^{53}$. No obstante, la Cumbre de Washington de 1999, junto con las conclusiones del Consejo Europeo de Niza del año 2000, así como con la declaración conjunta de diciembre de 2002, prepararon el camino para una estructura cooperativa militar sostenible entre la UE y la OTAN ${ }^{54}$. Los

${ }^{51}$ Actas de la sesión del Consejo de Seguridad de Naciones Unidas a propósito de la resolución 1487 (2003), 12 de junio. Doc. S/PV. 4772, p. 25.

${ }^{52}$ Idea en la que insiste ORENTLICHER, D. F., «Unilateral Multilateralism: United Sates Policy Toward the International Criminal Court», Cornell International Law Journal, 2004, p. 431.

${ }^{53}$ La primera misión militar de la UEO en Europa fue la misión de vigilancia en el Mar Adriático. También hubo cooperación en las operaciones posteriores de la UEO (operación de aplicación del embargo en el Danubio, apoyo a la administración de la ciudad de Mostar).

${ }^{54}$ Durante el Consejo Europeo de Sevilla de 2002, la diplomacia española intensificó sus esfuerzos para desbloquear las relaciones UE-OTAN, por cierto, por aquel entonces en crisis, y que constituyeron una gran contribución al hito histórico que supuso la Declaración conjunta sobre la asociación estratégica de ambas organizaciones. Estas acciones permitieron que unos meses más tarde, en marzo de 2003, se cerrara el acuerdo entre la OTAN y la UE. 
trabajos culminaron en los acuerdos Berlín Plus firmados el 17 de marzo del 2003, que constituyen un marco de colaboración permanente entre las dos organizaciones, y permitieron el acceso de la UE a medios y capacidades de la OTAN para el desempeño de sus operaciones de gestión de crisis.

Los acuerdos Berlin Plus pretendieron sincronizar las actividades de las dos organizaciones y evitar duplicidades en el ámbito estratégico militar. En particular, se acordaron temas relativos a los esquemas de consulta en épocas de crisis, mecanismos de desarrollo de capacidades, acceso a capacidades de planeamiento de la OTAN, acceso a medios y capacidades militares pre-identificados de la OTAN, determinación de una serie de opciones de mando OTAN (Cuarteles Generales), susceptibles de ser utilizados por la UE, así como la adaptación del sistema de planeamiento OTAN para contemplar las fuerzas disponibles para operaciones de la UE. Un elemento importante de los acuerdos Berlín Plus consistió en la integración de los Estados no miembros de la UE, pero miembros de la OTAN en las operaciones estratégicas de la UE. De esta manera, cuando la UE actúa sin el apoyo de la OTAN, los Estados no miembros de la UE pueden participar en las operaciones europeas si la UE aprueba su participación, mientras que en las operaciones que se desarrollan con la participación de la OTAN, los Estados no miembros de la UE pueden participar sin restricción alguna, si así lo desean.

De otro lado, los primeros lazos institucionales entre la UE y Naciones Unidas tuvieron lugar en el Consejo Europeo de Luxemburgo del año 2001, con la conclusión del Consejo sobre la cooperación UE-Naciones Unidas en materia de prevención de conflictos y gestión de crisis ${ }^{55}$. El Consejo Europeo convino los ámbitos específicos que constituirán el eje de los esfuerzos de la UE para intensificar la interrelaciónn con Naciones Unidas: prevención de conflictos; aspectos civiles y militares de la gestión de crisis, y problemas regionales concretos ${ }^{56}$. No obstante, las modalidades de la cooperación entre la UE y Naciones Unidas se redujeron en el documento a meras reuniones regulares (semestrales) entre altos representantes de Naciones Unidas (Secretario General y Secretario General Adjunto) y de la UE (Consejo de la Unión y Comisión).

La Declaración Conjunta del 24 de septiembre de 2003 sobre Cooperación entre Naciones Unidas y la UE en la gestión de crisis constituyó la base para la concentración de esfuerzos en apoyo a Naciones Unidas mediante la utilización de elementos civiles y militares de la PESD, así como mediante medidas conjuntas de formación ${ }^{57}$. Casi un año después de esta fecha, el Con-

55 «Cooperación UE-Naciones Unidas en materia de prevención de conflictos y gestión de crisis-Conclusiones del Consejo», Consejo de Asuntos Generales, Luxemburgo, 11 y 12 de junio de 2001 .

${ }^{56}$ Los Balcanes occidentales, Oriente Medio y África, en especial la región de los Grandes Lagos, el Cuerno de África y el África occidental.

${ }^{57}$ September 24, 2003: Joint Declaration on UN-EU Co-operation in Crisis Management (New York). 
sejo de la Unión publicó un Programa de Desarrollo de la Declaración Conjunta en el que se diseñaron posibles modelos de cooperación entre Naciones Unidas y la PESD que en esencia se redujeron a dos opciones: a) provisión de capacidades militares nacionales dentro del marco cooperativo con la ONU, $\mathrm{y}$ b) una operación de la UE como respuesta a una solicitud de la $\mathrm{ONU}^{58}$.

\section{CONCLUSIONES}

El análisis realizado en este estudio sobre el «modelo de gobernanza» de la política exterior de la UE es, ante todo, conceptual y descriptivo. El carácter distintivo de la «política exterior» de la UE con respeto a otros actores reside, como apunta la mayoría de la doctrina especializada, en que se trata de un actor internacional más fuerte y visible que el resto, y depende tanto de su limitada capacidad militar - en fase de construcción, hoy ralentizada-, como de sus principios y valores, e intereses. Desde luego, ambos elementos, valores, principios e intereses no se pueden disociar al analizar la política exterior de la UE. Quizás, y después del estudio realizado, uno de los elementos distintivos de la política exterior de la UE, como defiende Gráinne de Búrca $^{59}$, sea la aproximación a la gobernaza seguida por la UE. Ese modelo de gobernanza trata de ser estable, duradero en el tiempo, institucionalizado, y desarrollado en marcos jurídicos multilaterales en los que confluyen intereses y una amplia participación de actores para coordinar y regular la política externa.

En este punto, no hay que dejar de preguntarse si, de un lado, la política exterior de la UE es eficaz y, de otro, si es aplicable universalmente. En cuanto a la primera cuestión, es ampliamente aceptado y obvio que la UE es más eficaz globalmente como actor comerical y económico, que en el ámbito politico. La UE alcanza mayor visivilidad como actor global cuando trata de alcanzar intereses materiales (comerciales, finanacieros, económicos, migratorios, e inlcuso, de seguridad), que cuando persigue objetivos más difusos, y meficaz cuando promueve el «modelo de gobernanza» en marcos colectivos. Evaluar la eficacia del «modelo de gobernanza» de la UE requiere, en todo caso, de tiempo, sobre todo para identificar en qué campos ofrecería mejores resultados, de ser el caso.

En cuanto a la segunda cuestión, si es exportable el modelo de normas y principios de la UE, y en respuesta al análisis inductivo realizado en este estudio, el modelo de gobernanza de la UE está funcionando en diversos planos: América Latina, crisis financiera (FMI), seguridad y defensa, entre otros.

${ }^{58}$ EU-UN Co-operation in Military Crisis Management Operations Elements of Implementation of the EU-UN Joint Declaration Adopted by the European Council (17-18 June 2004).

${ }^{59}$ BÚRCA, G. DE, «EU External Relations: the Governance Mode of Foreign Policy», cit. supra nota 27. 
El carácter distintivo de la UE, como actor global, no es tanto por la promoción de principios y valores, y menos de intereses, o el recurso al derecho «blando» (soft-law) frente al derecho «duro» (hard-law), sino más bien por el «modelo de gobernanza» de la política exterior que promueve fundamentada en el consenso, y que es una suma de valores, principios, intereses, instrumentos, y mecanismos. En este contexto afloran, al menos, dos cuestiones más: de un lado, si el «modelo de gobernanza» de la política exterior de la UE es más eficaz cuando se enfrenta a problemas colectivos a nivel global que el promovido por otros actores (como es el caso de las negociaciones sobre el cambio climático), y de otro, si el «modelo de gobernanza» que aplica y promueve la UE es realmente colectivo y participatorio, o un instrumento que utiliza la UE para exportar sus reglas sustantivas y promover sus intereses particulares sin tener en cuenta las particularidades de los otros participantes. Bien podríamos encontrarnos con situaciones en los que la UE utiliza ambos extremos.

En todo caso, el TUE (2007) permite desarrollar la dimensión exterior del modelo europeo proponiendo y permitiendo que la UE impulse instituciones políticas a nivel global para la gestión multilateral de los problemas internacionales. El modelo a seguir por estas instituciones bien podría ser el europeo que ha experimentado con éxito las soberanías compartidas apoyada en los valores de la democracia, el pluralismo y el respeto a la diversidad. El TUE (2007) trata de contribuir a hacer gobernable la misteriosa globalización ${ }^{60}$, a regularla y a distribuir entre todos (al menos en ciertos supuestos), los beneficios de la misma, superando la doctrina de la suma de intereses nacionales y adoptando una política de responsabilidad en la gestión de los asuntos globales.

${ }^{60}$ KenNedy, D., «The Mystery of Global Governance», Ohio N.U.L. Rev., vol. 38, 2008, pp. 827-860. 